\title{
Thermal diffusivity and its lower bound in orthorhombic SnSe
}

\author{
Valentina Martelli ${ }^{1}$, Fabio Abud ${ }^{2}$, Julio Larrea Jiménez ${ }^{1}$, \\ Elisa Baggio-Saitovich ${ }^{3}$, Li-Dong Zhao ${ }^{4}$, and Kamran Behnia ${ }^{5}$ \\ (1) Laboratory for Quantum Matter under Extreme Conditions, \\ Instituto de Física, Universidade de São Paulo, \\ 05508-090, São Paulo, Brazil \\ (2) Instituto de Física, Universidade de São Paulo, \\ 05508-090, São Paulo, Brazil \\ (3) Centro Brasileiro de Pesquisas Física (CBPF) \\ (4) School of Materials Science and Engineering, \\ Beihang University, Beijing 100191, China \\ (5) Laboratoire de Physique et d'Étude des Matériaux \\ (ESPCI Paris - CNRS - Sorbonne Université), \\ PSL Research University, 75005 Paris, France
}

(Dated: July 16, 2021)

\begin{abstract}
The orthorhombic monochalcogenide SnSe has attracted much attention in recent years as a promising high-temperature thermoelectric material. We present a study of its thermal conductivity and specific heat of SnSe between $2 \mathrm{~K}$ and $300 \mathrm{~K}$ and quantify its anisotropic thermal diffusivity, $D$. For both crystallographic orientations, thermal diffusivity remains above the recently identified Planckian limit $\left(D>v_{s}^{2} \tau_{P}\right.$, where $v_{s}$ is the sound velocity and $\left.\tau_{P}=\hbar / k_{B} T\right)$ and its anisotropy in $D$ is set by the anisotropy of $v_{s}$. Comparison with cubic members of the IV-VI family leads to a consistent picture, where the diffusivity in all members of the family is set by the product of $\mathrm{v}_{s}, \tau_{P}$ and the 'melting' velocity derived from the melting temperature.
\end{abstract}

\section{INTRODUCTION}

Like elemental black phosphorus, SnSe crystallizes in an orthorhombic crystal structure. A member of groupIV monochalcogenides, it is a promising material with potential applications in fields ranging from solar cells and batteries to supercapacitors [1]. Its properties the monolayer limit are also attracting newt attention 208 . The bulk solid became a well-known thermoelectric material following the report of a large figure of merit driven by its low thermal conductivity [9 11]. The magnitude of thermal conductivity became a subject of controversy, with a large variety in its reported amplitudes [12 18.

SnSe is a layered solid with easy-cleaving $b-c$ planes in which atomic bonds are stronger than in the perpendicular orientation generating a significant anisotropy in structural properties. This orthorhombic crystal structure, found in other binary IV-VI salts (like GeSe) can be viewed as a distorted rock-salt cubic structure of $\mathrm{PbTe}$, another member of this family [19]. The competition between the rock-salt structure and the two (orthorhombic and rhombohedral) less symmetric options in this family is driven by an interplay between Peierls distortion, s-p hybridization and spin-orbit coupling and has been a longstanding subject of meditation, contemplation and debate [19 23].

Planckian time $\left(\tau_{p}=\hbar / k_{B} T\right.$, with $k_{B}$ Boltzmann constant and $T$ the absolute temperature) as a boundary to rate of dissipation has attracted much attention in recent years. In 2013, Bruin et al. 24] noticed that the effective scattering time of electrons in a variety of metals when electrical resistivity is linear in temperature is of the or- der of $\tau_{P}$. The behavior of phonons in insulators came under scrutiny a few years afterwards and it was found that when phonon-phonon scattering becomes dominant and thermal resistivity is $T$-linear, the phonon scattering time approaches $\tau_{P}$ without falling below it $[25,26$. This experimental observation raised a question. Above the Debye temperature, phonons obey the BoltzmannMaxwell distribution. Therefore, how can the Planck constant infer with their dissipation rate? Mousatov and Hartnoll 27. argued that this lower bound to thermal diffusivity is set by the energy scale of the melting temperature of the crystal. They proposed a universal scaling relation between the ratio of the phonon scattering time to the Planckian time and the ratio of the 'melting' velocity to the sound velocity.

The previous experimental observations 25, 26, 28 and the theoretical scenarios [27, 29] were focused on cubic insulators and ignored the issue of anisotropy. In this paper, we will present and examine the case of orthorhombic, and therefore anisotropic, SnSe. By quantifying the thermal diffusivity and sound velocity along the $b$ and $c$ directions, we will examine the account of the amplitude and anisotropy of thermal diffusivity in the Moussatov-Hartnoll picture.

\section{EXPERIMENTAL}

High-quality SnSe crystals were synthesized using a modified vertical Bridgman method 10. High-purity elemental constitutes were measured and loaded into carbon-coated conical silicon tubes and the crystals were grown in a temperature gradient from $1213 \mathrm{~K}$ to $973 \mathrm{~K}$ 
(a)
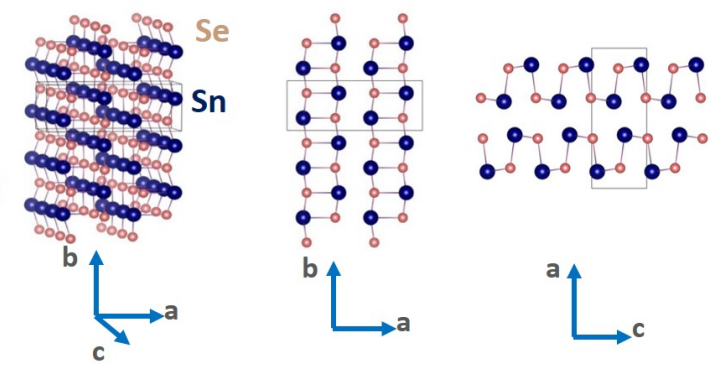

(b)

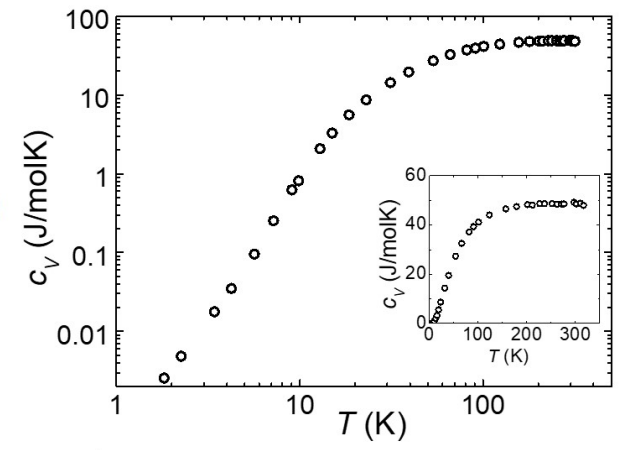

(c)

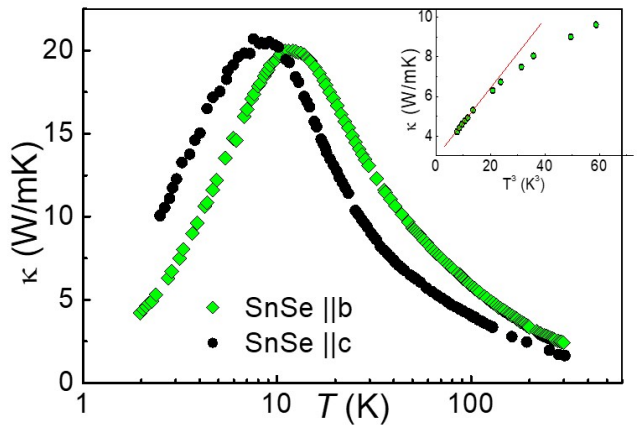

FIG. 1. Thermal conductivity and specific heat of SnSe: (a) The projections of the main crystallographic directions of the orthorhombic SnSe. Lattice parameters from Ref. 30. (b) Specific heat (2-300 K) of SnSe. The inset shows the same data set in a linear scale. (c) Thermal conductivity of SnSe as a function of temperature between $2-300 \mathrm{~K}$ when a thermal gradient is applied along the $b$ and $c$ directions.

at a slow rate of $1 \mathrm{~K} / \mathrm{h}$. Finally, SnSe crystals with diameter of $12 \mathrm{~mm}$ and length of $30 \mathrm{~mm}$ were obtained. The investigated single crystals display an $n$-type behaviour, with a carriers density in the order of $10^{16} \mathrm{~cm}^{-3}$. The contribution of charge carriers to thermal transport can be estimated by Wiedemann-Franz law and remains negligible in our range of measurements.

The specimens were aligned and cut appropriately to apply a thermal gradient along the in-plane $b$ and $c$ crystallographic directions, obtaining a geometric factor $g=\frac{S}{L}=1.5 \mathrm{~mm}$ where $S$ is the cross section area and $L$ is the separation between the leads that probe the temperature gradient. We measured thermal conductivity with a standard two-thermometers one-heater setup in the 2$300 \mathrm{~K}$ temperature range. Heat capacity was also determined between 2 and $300 \mathrm{~K}$, using a standard platform in a Quantum Design cryostat. The molar heat capacity

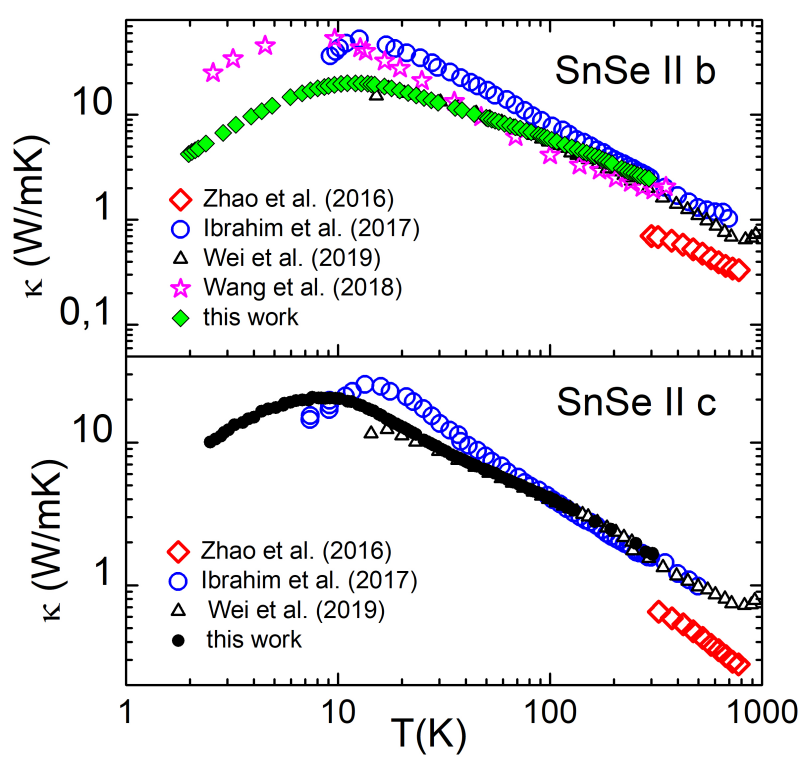

FIG. 2. Thermal conductivity of SnSe: Extended temperature range thermal conductivity of SnSe with heat conduction along the $b$ and $c$ direction, in the upper and lower panel respectively. Comparison between this work and previously reported data 9, 13, 14, 31.

is divided by the mass of the specimen and multiplied by the molar mass of SnSe, $M_{m}=197.67 \mathrm{~g} / \mathrm{mol}$. The density of the single crystals was determined by combining volume and mass measurements $\rho=(6.4 \pm 0.4) \mathrm{g} . \mathrm{cm}^{-3}$ that inside the experimental uncertainty is in agreement with the expected value for a fully dense sample.

\section{RESULTS}

Fig 1 (b) shows the temperature dependence of the specific heat data $C_{v}(T)$, to be compared with what was reported previously [9, 13, 15. We find that above $\sim$ $200 \mathrm{~K}$ the specific heat plateaus to a value of about $\sim 50$ J.K ${ }^{-1} . \mathrm{mol}^{-1}$, consistent with the expected Dulong-Petit value of $50 \mathrm{~J} . \mathrm{K}^{-1} \cdot \mathrm{mol}^{-1}$ for a solid with bi-atomic crystal cell. The Debye temperature $\theta_{D}$, determined by fitting the specific heat contribution $\beta$ in $C_{v} / T=\gamma+\beta T^{2}$ and quantifying $\beta$, was found to be $\theta_{D} \approx 190 \mathrm{~K}$, consistent with previous experimental data [15]. The theoretically expected value has been calculated to be $\theta_{D} \approx 140 \mathrm{~K}$ [32.

As seen in Fig]1(c), for both directions thermal conductivity displays the typical behaviour expected for an insulator. Thermal conductivity, $\kappa$, peaks at intermediate temperatures for both orientation. The peak height is about $20 \mathrm{~W} / \mathrm{m} . \mathrm{K}$ for both directions. The peak occurs at $12 \mathrm{~K}$ for $b$ direction and at $9 \mathrm{~K}$ for the $c$ direction. At low temperatures, below the peak, $\kappa$ displays a $T^{3}$ power law (see inset).

As seen in Fig. 2, according to our data, at room 
temperature, the amplitude of $\kappa$ is $2.4 \mathrm{~W} / \mathrm{m} . \mathrm{K}$ and $1.6 \mathrm{~W} / \mathrm{m} . \mathrm{K}$ along the $b$ and $c$ axes, respectively. A comparison of our data with previous publications indicates that our result is in good agreement with most works on single crystals [13, 14, 31, but differs by a factor 3 with respect to the reported ultra-low values 9 . The discrepancy among different data sets was debated previously 12. Our results on fully dense single crystalline samples reaffirm that likely the thermal conductivity of single crystalline SnSe are not as low as initially claimed and the discrepancy is a matter of accurate measurement.

Our experimental results can also be compared with Ab-initio calculations in both the low temperature orthorhombic phase $($ Pnma, $T<800$ T) 32 and in the high-temperature base-centered orthorhombic phase $(\mathrm{Cmcm}, \mathrm{T}>800 \mathrm{~T})$ [33. Since the structural phase transition is of second order, one expects a smooth evolution of the physical properties across it. Both theoretical works [32, 33] are in agreement with the results presented in this work and in other previous experimental studies [13, 31. This settles the magnitude of the intrinsic thermal conductivity in single-crystalline $\mathrm{SnSe}$ to be $\sim 2 \mathrm{~W} / \mathrm{m} . \mathrm{K}$ at room temperature.

The amplitude of the peak in $\kappa$ around $10 \mathrm{~K}$ varies between 20 and $60 \mathrm{~W} / \mathrm{m} . \mathrm{K}$, indicative of difference in the maximum phonon-mean-free-path across samples. At high temperature, thermal conductivity follows a $1 / T$ behavior with a clear anisotropy between the two crystallographic orientations, in agreement with what previously reported [13, 31.

Thermal diffusivity $D$ of a solid is defined by the heat equation $\frac{\partial T}{\partial t}=D \frac{\partial^{2} T}{\partial x^{2}}$, where thermal transport is supposed to be established along a certain direction (for instance $x$ ). Combining the Fourier equation with the conservation of energy, one finds that $D$ is the ratio of thermal conductivity and specific heat per volume. Fig. 3 (a) shows the obtained $D$ along $b$ and $c$ orientations. One can see that they cross around $10 \mathrm{~K}$. Above $100 \mathrm{~K}$, they display a $\sim T^{-1}$ behaviour. The phonon mean free path $(\lambda)$ along the two orientations is shown in fig. 3(b), extracted using $\kappa=\frac{1}{3} c_{p h} v_{s} \lambda$. The sound velocity $v_{s}$ for each direction was determined as discussed in the Appendix. At the lowest investigated temperature, $\lambda \sim 0.05 \mathrm{~mm}$, still more than one order of magnitude lower than the smallest geometrical dimension of the sample $(\sim 2 \mathrm{~mm})$, indicating that phonons do not reach the ballistic regime.

Fig. 4 compares our thermal diffusivity with previous studies of single crystalline SnSe and with three cubic members of IV-VI family. We can see that our results agree with the data reported by Ibrahim et al. [13].

\section{DISCUSSION}

It is instructive to compare the amplitude of the maximum $\kappa$ in SnSe and in black phosphorus, which are iso-structural. The $20-60 \mathrm{~W} / \mathrm{m}$.K peak in SnSe is more than one order of magnitude smaller than what was ob-
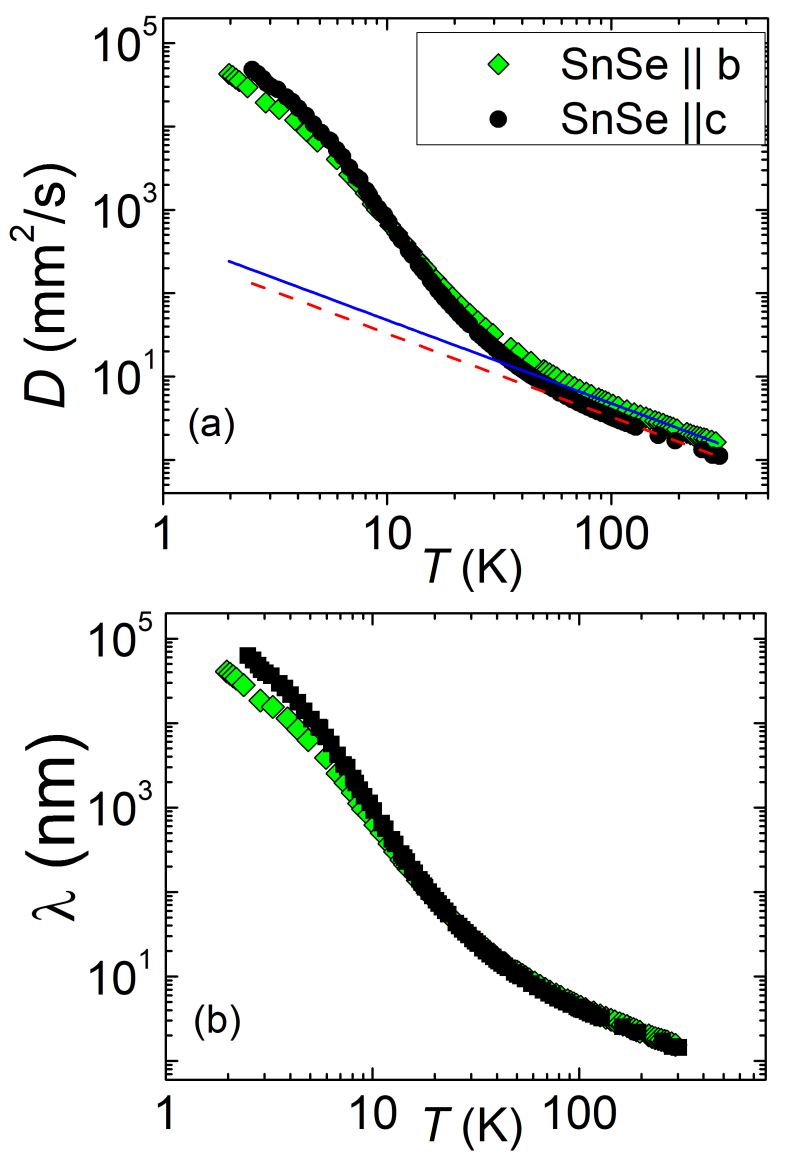

FIG. 3. Anisotropic diffusivity: (a) Thermal diffusivity and (b) mean free path of SnSe for $b$ and $c$ crystallographic directions. The solid and dashed lines in (a) represent $T^{-1}$ power law.

served black phosphorus 34. In the latter system, below the peak temperature, the phonon mean-free-path is comparable to the thickness and the amplitude of the thermal conductivity is size-dependent 34. In contrast, in $\mathrm{SnSe}$, in the ballistic regime is not attained. This implies the presence of disorder spatially extended enough to scatter long wavelength phonons. The absence of ballistic phonon transport hinders the possible emergence of phonon hydrodynamics [35] driven by abundance of momentum-conserving phonon-phonon collisions 25, 34, 36.

We now turn our attention to the thermal diffusivity. The existence of a lower bound to thermal diffusivity $D$ in insulators [25, 26] was noticed through the scrutiny of the slope of $D$ as a function of inverse of temperature in various solids. The phonon scattering time $\tau$ can be extracted, using:

$$
D=v_{s}^{2} \tau
$$

It was found that even in the least conducting known materials $\tau$ approaches, but does not fall below the Planck- 
(a)

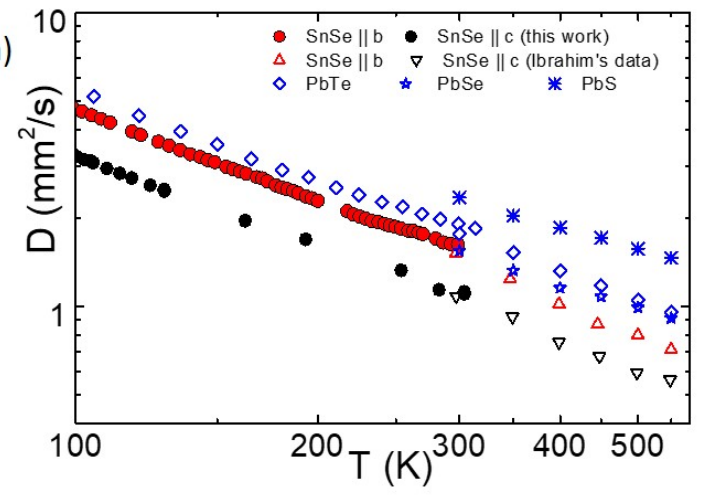

(b)

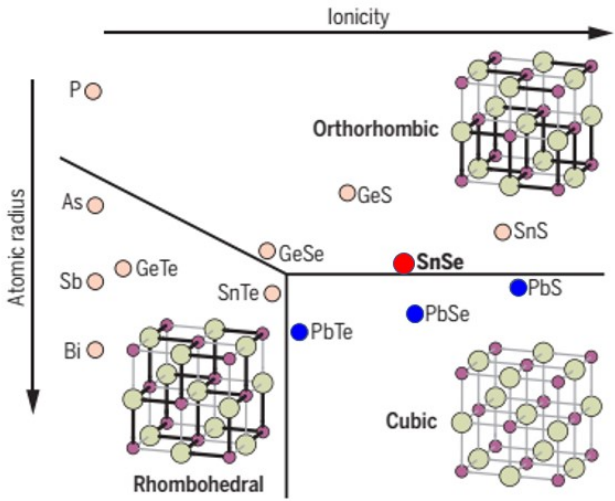

FIG. 4. High-temperature diffusivity: (a) Hightemperature diffusivity of SnSe compared with other calchogenides. Values for $\mathrm{PbTe}, \mathrm{PSe}$ and $\mathrm{PbS}$ are obtained from [37 [40; SnSe [13]. (b) Structural phase diagram of IV-VI salts (adapted from Ref. [19, 23]).

ian time $\tau_{p}$ :

$$
\tau=s \tau_{p}=s\left[\frac{\hbar}{k_{B} T}\right]
$$

where $s$ is a material-dependent constant that was found to be larger than unity in all known materials.

Mousatov and Hartnoll 27. proposed that the proximity to the Planckian limit is controlled by the sound velocity $v_{s}$ constrained by the bound $v_{s} \leq v_{M}$, where $v_{M}$ is the crystal melting velocity. The latter is defined as $v_{M} \equiv \frac{k_{B} T_{M}}{\hbar} a$ where $T_{M}$ is the melting temperature and $a$ is the lattice spacing. The closer is $v_{s}$ to $v_{M}$, the closer is a certain the material to the lower bound. The melting velocity defined by the melting temperature and the atomic spacing sets an upper boundary to the velocity in a crystal. They showed that for a large number of compounds $\tau / \tau_{p}$ and $v_{M} / v_{s}$ display a roughly linear correlation [27. More recently, Xu et al. 28 measured the thermal conductivity and diffusivity of cubic $\operatorname{In}_{2} \mathrm{O}_{3}$ and found that the properties of this compound fit in this picture.

The case of orthorhombic SnSe provides an opportunity to probe the role of the anisotropy of the sound velocity in this picture. Using the components of the elastic tensor experimentally reported in ref. 41], we calculated the longitudinal sound velocities along $b$ and $c$ to be, respectively $v_{l, b}=3146 \mathrm{~m} / \mathrm{s}$ and $v_{l, c}=2317 \mathrm{~m} / \mathrm{s}$ (see Appendix). Remarkably, the ratio of the velocities along the two orientation corresponds to the anisotropy of thermal diffusivity in the intrinsic regime. Both thermal diffusivity and sound velocity are roughly 1.3 times larger along the b-axis compared to the c-axis.

We note that the amplitude of sound velocity is compatible with the moderate atomic mass of atoms in SnSe. According to a recent expression for the speed of sound $v_{s}=\frac{\alpha}{c} \sqrt{\left(m_{e} / 2 M\right)}$ [42], where $c$ is the light velocity, $\alpha$ is the fine structure constant and $m_{e}$ and $M$ are the masses of bare electron and the atom, respectively. Injecting the mass of Sn (119 atomic mass units), one finds $3500 \mathrm{~m} / \mathrm{s}$.

Taking the high temperature slope of the diffusivity $D \cdot k_{B} /\left(\hbar v_{s}^{2}\right)$ as a function of $T^{-1}$ (see straight lines in Fig. 3 (a)) one can quantify the parameter $s$ for both directions considering the computed value for the directiondependent sound velocity $v_{s}$. We found that $s_{\| b}=6.3$ and $s_{\| c}=8$. We did not measure thermal conductivity along the a-axis, but using the data reported by Ibrahim et al., one obtains $s_{\| a}=4$.

The phonon lifetime according to Raman spectroscopy is $\tau \sim 0.1 \mathrm{ps}$ at $800 \mathrm{~K}[43$; using this time, one finds $\tau / \tau_{P} \sim 10$ in good agreement with what is found here.

The melting velocity $v_{M}$ of SnSe can be calculated from the melting temperature $T_{M}=1134 \mathrm{~K}$ [4] and the average interatomic distance $a=\sqrt[3]{\frac{M_{m}}{N_{A} n_{m}}}$ where $M_{m}$ is the molar mass $(197.67 \mathrm{~g} / \mathrm{mol}), N_{A}$ is the Avogadro number and $n_{m}=2$ is the number of atoms per unit formula in SnSe. We obtain $v_{M}=44658 \mathrm{~m} / \mathrm{s}$ for SnSe. This allows us to put SnSe on Fig. 5 (re-adapted from Ref. [28]) together with a large number of systems previously tabulated [27. One can see that along b-axis, SnSe is close to the cubic members of the IV-VI family. The larger $s$ for the c-axis corresponds can be explained by a lower sound velocity along this direction, which amplifies both $\tau / \tau_{p}$ and $v_{M} / v_{s}$.

Let us note that $\frac{D}{v_{s}^{2} \tau_{P}} \propto \frac{v_{M}}{v_{s}}$ can be rewritten as $\frac{D}{v_{s}} \propto \tau_{P} v_{M}$. The right hand side is isotropic implying that the anisotropy of $D$ and $v_{s}$ should cancel out. As one can see in figure 5, for the 4 members of the IV-VI family examined here, the proportionality factor is close to 0.5 . As a consequence, the expression $D \approx 0.5 v_{s} v_{M} \tau_{P}$ gives a satisfactory account of the magnitude of $D$ for both orientations in SnSe as well as the cubic members of this family, confirming the relevance of the picture put forward in ref. 27] to members of this family.

The anisotropy of thermal diffusivity appears to be driven by the anisotropy of sound velocity. The intrinsic phonon anharmonicity due to the proximity of a loweringsymmetry structural transition [17, 19, 23. plays a major role. Moreover, sound velocity depends on the elastic constants, whose link to phonon anharmonicity was discussed previously 45. Recently, Wu and Sau 46] have proposed a heat transport model in a strongly anhar- 


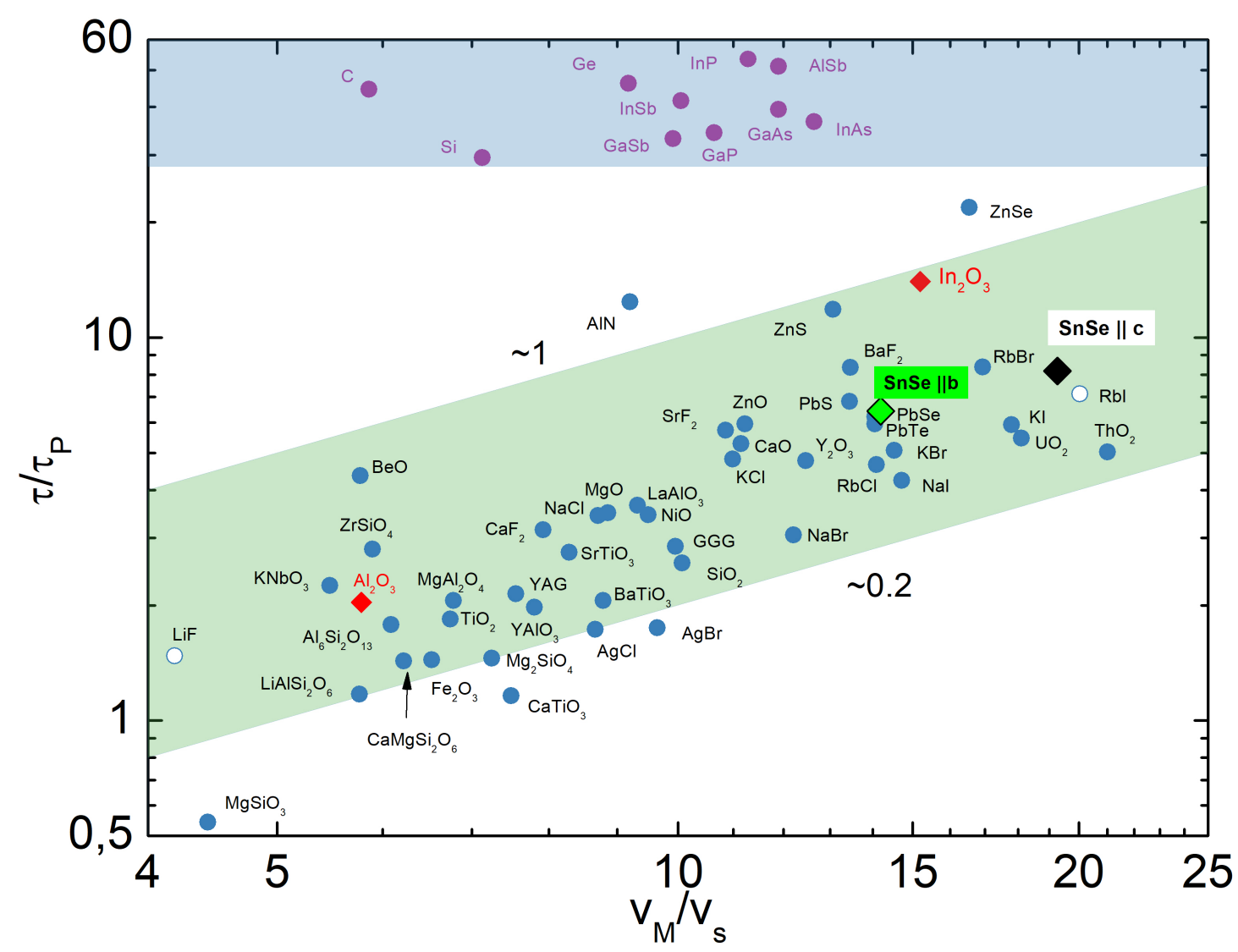

FIG. 5. Scattering time vs sound velocity: $\tau / \tau_{p}$ vs $v_{M} / v_{s}$ for different materials (Figure adapted from Ref. [28]) compared with SnSe data of this work. The new points of SnSe are determined taking into account, as the $v_{s}$, the longitudinal sound velocity for each direction.

monic system in which the thermal diffusivity can become lower than the Planckian bound. Our results indicate that SnSe in spite of its low thermal diffussivity and strong anharmoinicity respects the Planckian bound.

We end our discussion by comparing the magnitude of thermal diffusivity in this solid with the Universal lower bounds to thermal diffusivity of liquids 47]. If $D$ decreases linearly down to the melting temperature of 1138 $\mathrm{k}$, it will become as small as $\approx 0.2 \mathrm{~mm}^{2} / \mathrm{s}$ at the onset of melting. This is still one order of magnitude larger than the lower boundary $\left(D_{\min }=\frac{1}{4 \pi} \frac{\hbar}{m_{e} M}\right)$ [47] in a supercritical fluid with an atomic mass of $M \approx 100$ amu.

In summary, we studied the anisotropic thermal diffusivity of SnSe for two crystallographic orientations between the $2 \mathrm{~K}$ and $300 \mathrm{~K}$. We found that our experimental findings matches a recent theoretical framework provided that one takes into account the anisotropy of sound velocity.

VM acknowledges the support of JP-FAPESP (2018/19420-3). JLJ acknowledges support of JPFAPESP (2018/08845-3) and of CNPq-Universal (431083/2018-5). This work has also been supported by FAPESP (2019/26141-6). KB is supported by the Agence Nationale de la Recherche (ANR-18-CE92-
0020-01; ANR-19-CE30-0014-04). EBS acknowledges supports from FAPERJ under PVE E-26/101.468/2014, E-26/010.002990/2014 and E-261010.001246/201.

\section{A. APPENDIX}

In this appendix we recall the relation between longitudinal velocity and elastic moduli, in particular for the case of an orthorhombic crystal structure.

In a generic crystal, for a certain crystallographic direction, we can define three sound velocities (one longitudinal and two transverse), whose description is a function of the stress tensor [48]. In a Cartesian representation, 9 stress components acting on the elementary cell can be generally considered: $X_{x}, X_{y}, X_{z}, Y_{x}, Y_{y}, Y_{z}, Z_{x}$, $Z_{y}, Z_{z}$. The capital letters point to the direction of the force, whereas the index indicates the direction which is perpendicular to the area where the force acts. As the total torque acting on the elementary cell has to be zero, the independent conditions are 6 as $Y_{z}=Z_{y}, Z_{x}=X_{z}$, $X_{y}=Y_{x}$.

In response to that external stress, the displacement of 
the deformation can be described by the strain $R(r)$ :

$$
R(r)=u(\mathbf{r}) \hat{x}+v(\mathbf{r}) \hat{y}+w(\mathbf{r}) \hat{z}
$$

where $u(\mathbf{r}), v(\mathbf{r})$ and $w(\mathbf{r})$ represent the displacements along the $x, y$ and $z$ axes, respectively. We define the strain components by the following relations:

$$
\begin{gathered}
e_{x x} \equiv \frac{\partial u}{\partial x} \quad e_{y y} \equiv \frac{\partial v}{\partial y} \quad e_{z z} \equiv \frac{\partial w}{\partial z} \\
e_{x y} \equiv \frac{\partial u}{\partial y}+\frac{\partial v}{\partial x} e_{y z} \equiv \frac{\partial v}{\partial z}+\frac{\partial w}{\partial y} \quad e_{x y} \equiv \frac{\partial u}{\partial z}+\frac{\partial w}{\partial x}
\end{gathered}
$$

For small deformation, we can apply the Hooke's law and relate, at first approximation, the force (stress) to the strain through the elastic moduli $C_{\alpha \beta}$. For instance, for the $X_{x}$ stress component:

$X_{x}=C_{11} e_{x x}+C_{12} e_{y y}+C_{13} e_{z z}+C_{14} e_{y z}+C_{15} e_{z x}+C_{16} e_{x y}$

The $36 C_{\alpha \beta}$ elastic constants, obtained when eq. $\mathrm{A} 4$ is written for all directions, reduce to 21 , as it can be shown that $C_{\alpha \beta}=C_{\beta \alpha}$.

When the crystal symmetry is taken into account, the number of non-zero elastic constants can be reduced to 3 for a cubic system, 5 for an hexagonal system, and 9 for an orthorhombic. The latter is the case of interest in this work.

The sound velocities of SnSe can be computed through the 9 non-zero elastic moduli of the stiffness tensor (see Table I), which were measured by ultrasound spectroscopy in single crystalline SnSe [41].

\begin{tabular}{c|cccccc} 
& $e_{x x}$ & $e_{y y}$ & $e_{z z}$ & $e_{y z}$ & $e_{z x}$ & $e_{x y}$ \\
\hline$X_{x}$ & $C_{11}$ & $C_{12}$ & $C_{13}$ & 0 & 0 & 0 \\
$Y_{y}$ & $C_{21}$ & $C_{22}$ & $C_{23}$ & 0 & 0 & 0 \\
$Z_{z}$ & $C_{31}$ & $C_{32}$ & $C_{33}$ & 0 & 0 & 0 \\
$Y_{z}$ & 0 & 0 & 0 & $C_{44}$ & 0 & 0 \\
$Z_{x}$ & 0 & 0 & 0 & 0 & $C_{55}$ & 0 \\
$X_{y}$ & 0 & 0 & 0 & 0 & 0 & $C_{66}$
\end{tabular}

TABLE I. Elastic constants: The non-zero constant for an orthorhombic system. Numerical values are obtained from Ref. [41] and expressed in GPa: $C_{11}=41.8, C_{22}=59.7$, $C_{33}=32.4, C_{44}=13.2, C_{55}=24.5, C_{66}=20.5, C_{12}=3.15$, $C_{13}=10.7, C_{23}=26.8$.

Then, we can relate the resultant force with the displacement, writing the motion equation. For instance, let us consider a travelling wave along [100], which corresponds to what was dubbed $x$-direction.

$$
u(\mathbf{r})=u_{0} \exp [i(k x-\omega t)]
$$

The motion equation is:

$$
\rho \frac{\partial^{2} u}{\partial t^{2}}=\frac{\partial X_{x}}{\partial x}+\frac{\partial X_{y}}{\partial y}+\frac{\partial X_{z}}{\partial z}
$$

and a similar expression is found for $v(\mathbf{r})$ and $w(\mathbf{r})$.

Let us substitute $u(\mathbf{r})$ in equation $\mathrm{A} 6$ ).

$$
\omega^{2} \rho=C_{11} k^{2}
$$

that leads to the the longitudinal velocity:

$$
v_{l x}=\frac{\omega}{k}=\sqrt{\frac{C_{11}}{\rho}}
$$

Considering instead a transverse displacement $(v$ or $w)$, with the wave propagating again along the $x$ direction, $v=v_{0} \exp [i(k x-\omega t)]$, we can obtain the transverse velocity.

With an analogous approach, the second transverse velocity can be obtained. Again, applying the calculation to the other two directions and solving the motion equations, we can complete table III. Finally, we can compute sound velocities for SnSe using the available elastic constants (Table III). The longitudinal velocities found here are used in the main text for the $b$ and $c$ crystallographic directions.

\begin{tabular}{||c|c|c|c||}
\hline $\begin{array}{c}\text { Wave } \\
\text { direction }\end{array}$ & $\begin{array}{c}\text { Longitudinal } \\
\text { velocity }\end{array}$ & $\begin{array}{c}\text { I-Transverse } \\
\text { velocity }\end{array}$ & $\begin{array}{c}\text { II-Transverse } \\
\text { velocity }\end{array}$ \\
\hline \hline $\mathrm{x}$ & $\sqrt{\frac{C_{11}}{\rho}}$ & $v_{x y}=\sqrt{\frac{C_{66}}{\rho}}$ & $v_{x z}=\sqrt{\frac{C_{55}}{\rho}}$ \\
\hline $\mathrm{y}$ & $\sqrt{\frac{C_{22}}{\rho}}$ & $v_{y x}=\sqrt{\frac{C_{66}}{\rho}}$ & $v_{y z}=\sqrt{\frac{C_{44}}{\rho}}$ \\
\hline $\mathrm{z}$ & $\sqrt{\frac{C_{33}}{\rho}}$ & $v_{z x}=\sqrt{\frac{C_{55}}{\rho}}$ & $v_{z y}=\sqrt{\frac{C_{44}}{\rho}}$ \\
\hline
\end{tabular}

TABLE II. Sound velocities: Anisotropic sound velocities for an orthorhombic structure.

\begin{tabular}{|c|c|c|c|}
\hline Axis & $\begin{array}{c}v_{l} \\
{[m / s]}\end{array}$ & $\begin{array}{c}v_{t 1} \\
{[m / s]}\end{array}$ & $\begin{array}{c}v_{t 2} \\
{[m / s]}\end{array}$ \\
\hline$a$ & 2632 & 1843 & 2015 \\
\hline$b$ & 3146 & 1843 & 1479 \\
\hline$c$ & 2317 & 2015 & 1479 \\
\hline
\end{tabular}

TABLE III. SnSe sound velocities: Longitudinal and transverse sound velocities of SnSe calculated starting from the elastic constant elements, along the main crystallographic axes.

The average sound velocity, which is often employed, can be related to the elastic moduli. Nevertheless, it is noteworthy to observe that this implies an approximation that we recall here below.

The average sound velocity can be determined from the following formula [49]:

$$
v_{s}=\left(\frac{1}{3} \sum_{i=1}^{3} \int_{V} \frac{1}{v_{i}^{3}} \frac{d \Omega}{4 \pi}\right)^{-1 / 3}
$$

where the integral considers the three velocities (longitudinal and two transverse) for a large number of directions 
in the space. The expression simplifies in the frequently used equation:

$$
v_{s}=\left(\frac{1}{3}\left[\frac{2}{v_{t}^{3}}+\frac{1}{v_{l}^{3}}\right]\right)
$$

that strictly holds only when the material is isotropic, which means when transverse $\left(v_{t}\right)$ and longitudinal sound velocities $\left(v_{l}\right)$ are invariant with the direction. The latter condition applies for instance in polycrystalline materials or isotropic glasses. In 1963, it was shown that a good approximation of the average sound velocity can be ontained through the so called VRHG approximation (Voigt, Reuss, Hill and Gilvarry), which establishes a criterium to connect the elastic tensor determined for a single crystal with the average shear and bulk moduli determined for the same compounds but in the polycristalline form [50]. There, average transverse $\left(\bar{v}_{t}\right)$ and longitudinal $\left(\bar{v}_{l}\right)$ velocities are defined according to: $\bar{v}_{t}=\sqrt{G / \rho}$ and $\bar{v}_{l}=\sqrt{B / \rho}$, where $B$ and $G$ are respectively the Hill's average bulk and shear moduli of a polycristal isotropic sample that are obtained as a function of the stress tensor's components of a single crystal of the same compound: $B=B\left(C_{i j}\right)$ and $B=B\left(S_{i j}\right)$ [41, 50]. $C_{i j}$ are the elastic constants and $S_{i j}=C_{i j}^{-1}$ are the elastic compliance constants. The elements of this tensor can be experimentally determined and so the average velocity $\bar{v}_{m}=\left(\frac{1}{3}\left[\frac{2}{\bar{v}_{s}^{3}}+\frac{1}{\bar{v}_{l}^{3}}\right]\right) . \quad \bar{v}_{s}$ was shown to approximate closely $v_{s}$ for a large class of materials. Applying this recipe, the average sound velocity for polycrystalline SnSe can be calculated from the elastic moduli: $1803 \mathrm{~m} / \mathrm{s}$ as in [4]. It is important to observe that in SnSe it exists a significant difference among the average sound velocities and the direction-dependent sound velocities.
[1] M. Kumar, S. Rani, Y. Singh, K. S. Gour, and V. N. Singh, Tin-selenide as a futuristic material: properties and applications, RSC Adv. 11, 6477 (2021)

[2] S. Barraza-Lopez, B. M. Fregoso, J. W. Villanova, S. S. Parkin, and K. Chang, Colloquium: Physical properties of group-IV monochalcogenide monolayers, Reviews of Modern Physics 93, 011001 (2021).

[3] Y. Huang, L. Li, Y.-H. Lin, and C.-W. Nan, Liquid exfoliation few-layer SnSe nanosheets with tunable band gap, The Journal of Physical Chemistry C 121, 17530 (2017).

[4] L. C. Gomes and A. Carvalho, Phosphorene analogues: Isoelectronic two-dimensional group-IV monochalcogenides with orthorhombic structure, Physical Review B 92, 085406 (2015).

[5] H. Wang and X. Qian, Two-dimensional multiferroics in monolayer group IV monochalcogenides, 2D Materials 4, 015042 (2017).

[6] M. Wu and X. C. Zeng, Intrinsic ferroelasticity and/or multiferroicity in two-dimensional phosphorene and phosphorene analogues, Nano letters 16, 3236 (2016).

[7] R. Fei, W. Li, J. Li, and L. Yang, Giant piezoelectricity of monolayer group IV monochalcogenides: SnSe, SnS, GeSe, and GeS, Applied Physics Letters 107, 173104 (2015).

[8] L. V. Titova, B. M. Fregoso, and R. L. Grimm, GroupIV monochalcogenides GeS, GeSe, SnS, SnSe, in Chalcogenide (Elsevier, 2020) pp. 119-151.

[9] L.-D. Zhao, S.-H. Lo, Y. Zhang, H. Sun, G. Tan, C. Uher, C. Wolverton, V. P. Dravid, and M. G. Kanatzidis, Ultralow thermal conductivity and high thermoelectric figure of merit in SnSe crystals, Nature 508, 373 (2014).

[10] C. Chang, M. Wu, D. He, Y. Pei, C.-F. Wu, X. Wu, H. Yu, F. Zhu, K. Wang, Y. Chen, et al., 3D charge and $2 \mathrm{D}$ phonon transports leading to high out-of-plane ZT in n-type SnSe crystals, Science 360, 778 (2018).

[11] C. Chang, G. Tan, J. He, M. G. Kanatzidis, and L.-D. Zhao, The thermoelectric properties of SnSe continue to surprise: extraordinary electron and phonon transport, Chemistry of Materials 30, 7355 (2018).

[12] P.-C. Wei, S. Bhattacharya, J. He, S. Neeleshwar,
R. Podila, Y. Chen, and A. Rao, The intrinsic thermal conductivity of SnSe, Nature 539, E1 (2016).

[13] D. Ibrahim, J.-B. Vaney, S. Sassi, C. Candolfi, V. Ohorodniichuk, P. Levinsky, C. Semprimoschnig, A. Dauscher, and B. Lenoir, Reinvestigation of the thermal properties of single-crystalline SnSe, Applied Physics Letters 110, 032103 (2017).

[14] S. Wang, S. Hui, K. Peng, T. P. Bailey, W. Liu, Y. Yan, X. Zhou, X. Tang, and C. Uher, Low temperature thermoelectric properties of p-type doped single-crystalline SnSe, Applied Physics Letters 112, 142102 (2018).

[15] S. Sassi, C. Candolfi, J.-B. Vaney, V. Ohorodniichuk, P. Masschelein, A. Dauscher, and B. Lenoir, Assessment of the thermoelectric performance of polycrystalline ptype SnSe, Applied Physics Letters 104, 212105 (2014).

[16] F. Serrano-Sánchez, M. Gharsallah, N. Nemes, F. Mompean, J. Martínez, and J. Alonso, Record Seebeck coefficient and extremely low thermal conductivity in nanostructured SnSe, Applied Physics Letters 106, 083902 (2015).

[17] C. W. Li, J. Hong, A. F. May, D. Bansal, S. Chi, T. Hong, G. Ehlers, and O. Delaire, Orbitally driven giant phonon anharmonicity in SnSe, Nature Physics 11, 1063 (2015).

[18] J. P. Heremans, The anharmonicity blacksmith, Nature Physics 11, 990 (2015).

[19] P. B. Littlewood, The crystal structure of IV-VI compounds. I. classification and description, Journal of Physics C: Solid State Physics 13, 4855 (1980).

[20] J. K. Burdett, From bonds to bands and molecules to solids, Progress in Solid State Chemistry 15, 173 (1984)

[21] D.-K. Seo and R. Hoffmann, What determines the structures of the group 15 elements?, Journal of Solid State Chemistry 147, 26 (1999).

[22] R. Peierls, More Surprises in Theoretical Physics (Princeton University Press, 1991).

[23] K. Behnia, Finding merit in dividing neighbors, Science 351, 124 (2016).

[24] J. Bruin, H. Sakai, R. Perry, and A. Mackenzie, Similarity of scattering rates in metals showing T-linear resistivity, Science 339, 804 (2013). 
[25] V. Martelli, J. L. Jiménez, M. Continentino, E. BaggioSaitovitch, and K. Behnia, Thermal transport and phonon hydrodynamics in strontium titanate, Physical review letters 120, 125901 (2018).

[26] K. Behnia and A. Kapitulnik, A lower bound to the thermal diffusivity of insulators, Journal of Physics: Condensed Matter 31, 405702 (2019).

[27] C. H. Mousatov and S. A. Hartnoll, On the Planckian bound for heat diffusion in insulators, Nature Physics 16, 579 (2020).

[28] L. Xu, B. Fauqué, Z. Zhu, Z. Galazka, K. Irmscher, and K. Behnia, Thermal conductivity of bulk $\operatorname{In}_{2} \mathrm{O}_{3}$ single crystals, Physical Review Materials 5, 014603 (2021).

[29] E. Tulipman and E. Berg, Strongly coupled quantum phonon fluid in a solvable model, Phys. Rev. Research 2, $033431(2020)$

[30] T. Chattopadhyay, J. Pannetier, and H. Von Schnering, Neutron diffraction study of the structural phase transition in SnS and SnSe, Journal of Physics and Chemistry of Solids 47, 879 (1986).

[31] P.-C. Wei, S. Bhattacharya, Y.-F. Liu, F. Liu, J. He, Y.H. Tung, C.-C. Yang, C.-R. Hsing, D.-L. Nguyen, C.-M. Wei, et al., Thermoelectric figure-of-merit of fully dense single-crystalline SnSe, ACS omega 4, 5442 (2019).

[32] J. Carrete, N. Mingo, and S. Curtarolo, Low thermal conductivity and triaxial phononic anisotropy of SnSe, Applied Physics Letters 105, 101907 (2014).

[33] U. Aseginolaza, R. Bianco, L. Monacelli, L. Paulatto, M. Calandra, F. Mauri, A. Bergara, and I. Errea, Phonon collapse and second-order phase transition in thermoelectric SnSe, Physical review letters 122, 075901 (2019).

[34] Y. Machida, A. Subedi, K. Akiba, A. Miyake, M. Tokunaga, Y. Akahama, K. Izawa, and K. Behnia, Observation of poiseuille flow of phonons in black phosphorus, Science advances 4, eaat3374 (2018).

[35] H. Beck, P. F. Meier, and A. Thellung, Phonon hydrodynamics in solids, Physica Status Solidi (a) 24, 11 (1974).

[36] Y. Machida, N. Matsumoto, T. Isono, and K. Behnia, Phonon hydrodynamics and ultrahigh-roomtemperature thermal conductivity in thin graphite, Science 367, 309 (2020).

[37] D. Parkinson and J. Quarrington, The molar heats of lead sulphide, selenide and telluride in the temperature range $20 \mathrm{~K}$ to $260 \mathrm{~K}$, Proceedings of the Physical Society. Section A 67, 569 (1954).
[38] A. Pashinkin, M. Mikhailova, A. Malkova, and V. Fedorov, Heat capacity and thermodynamic properties of lead selenide and lead telluride, Inorganic Materials 45, 1226 (2009).

[39] A. El-Sharkawy, A. Abou El-Azm, M. Kenawy, A. Hillal, and H. Abu-Basha, Thermophysical properties of polycrystalline $\mathrm{PbS}, \mathrm{PbSe}$, and $\mathrm{PbTe}$ in the temperature range 300-700K, International journal of thermophysics 4, 261 (1983).

[40] D. Morelli, V. Jovovic, and J. Heremans, Intrinsically minimal thermal conductivity in cubic I-V-VI2 semiconductors, Physical review letters 101, 035901 (2008).

[41] A. Karunarathne, J. R. Gladden, G. Priyadarshan, P.C. Wei, T.-L. Hung, P. Parajuli, S. Bhattacharya, Y.Y. Chen, and A. M. Rao, Room temperature resonant ultrasound spectroscopy of single crystalline SnSe, ACS Applied Energy Materials 1, 6123 (2018).

[42] K. Trachenko, B. Monserrat, C. Pickard, and V. Brazhkin, Speed of sound from fundamental physical constants, Science Advances 6, eabc8662 (2020).

[43] F. Liu, P. Parajuli, R. Rao, P. Wei, A. Karunarathne, S. Bhattacharya, R. Podila, J. He, B. Maruyama, G. Priyadarshan, et al., Phonon anharmonicity in singlecrystalline SnSe, Physical Review B 98, 224309 (2018).

[44] H. Okamoto, Se-Sn (selenium-tin), Journal of phase equilibria 19, 293 (1998).

[45] A. Karunarathne, P. Parajuli, G. Priyadarshan, S. Bhattacharya, R. Rao, P.-C. Wei, Y.-Y. Chen, J. R. Gladden, and A. M. Rao, Anisotropic elasticity drives negative thermal expansion in monocrystalline SnSe, Physical Review B 103, 054108 (2021).

[46] H.-K. Wu and J. D. Sau, Classical model for subplanckian thermal diffusivity in complex crystals, Phys. Rev. B 103, 184305 (2021).

[47] K. Trachenko, M. Baggioli, K. Behnia, and V. V. Brazhkin, Universal lower bounds on energy and momentum diffusion in liquids, Phys. Rev. B 103, 014311 (2021)

[48] C. Kittel, Introduction to Solid State Physics. [With Illustrations.]. (John Wiley \& Sons, 1966).

[49] H. B. Huntington, The elastic constants of crystals, Solid state physics 7, 213 (1958).

[50] O. L. Anderson, A simplified method for calculating the Debye temperature from elastic constants, Journal of Physics and Chemistry of Solids 24, 909 (1963). 